\title{
28 Research Soure \\ Expression of MMP28 in bladder cancer tissue microarray and its clinical significance
}

\section{Wang Heng}

Bengbu Medical College https://orcid.org/0000-0003-1485-7032

Wu Junxiu

Zhejiang Chinese Medical University

Chen Xinpeng

Bengbu Medical College

Zhang Qi

Zhejiang Provincial People's Hospital

Zhao Tingxiao

Bengbu Medical College

Zhang Dahong ( $\square$ clinic@126.com )

\section{Research article}

Keywords: Bladder cancer, MMP28, prognostic marker, prognosis

Posted Date: August 14th, 2019

DOl: https://doi.org/10.21203/rs.2.12805/v1

License: (c) (1) This work is licensed under a Creative Commons Attribution 4.0 International License.

Read Full License 


\section{Abstract}

Background: The aim of this study was to explore the expression pattern and prognostic value of MMP28 for bladder cancer and analyze its relationship with the clinicopathological features of human bladder cancer. Methods: Immunohistochemical staining for MMP28 was performed in 491 archived radical bladder cancer resection and 80 normal specimens. The immunoreactivity of these proteins was correlated to evaluate their clinical significance as prognostic factors. Results: Protein level of MMP-28 was up-regulated in bladder cancer compared with adjacent non-tumor tissues. The increased expression of MMP-28 was significantly associated with high histological grade, lymph node metastasis, lymphatic invasion and distant metastasis $(\mathrm{P}<0.05)$. High expression of MMP-28 was also associated with greater risk of disease progression and decreased chance of cancer-specific survival. Further analysis suggested that MMP-28 was related with decreased overall survival. Conclusions: MMP-28 could be used as an effective marker for tumor diagnosis and predict tumor progression in bladder cancer. The expression patterns of MMP-28 interaction correlated well with the pathological stage, disease progression and tumor-specific survival. The finding may help identify more biologically aggressive carcinomas which could aid in patients who benefit from more intensive adjuvant therapy.

\section{Background}

Bladder cancer $(\mathrm{BC})$ is the most common malignant tumors of the urinary tract, and the incidence of $\mathrm{BC}$ is gradually increasing. Recently, it has become the fourth most common malignancy in man and the ninth in female $(1,2)$. In fact, the early stage of bladder cancer often lacks obvious symptoms, whereas the advanced stage of it is highly metastatic, which leads to a high mortality rate of the disease $(3,4)$. The development of bladder cancer is a complex pathological process, it can improve the difficulty of treatment. Current treatment methods for bladder cancer mainly including: surgical treatment, chemotherapy, radiotherapy, and combined immunotherapy. Especially radical cystectomy, as a standard treatment method for muscle-invasive bladder cancer, has been widely used in clinical treatment, but nearly half of these patients developed metastasis within two years of cystectomy and subsequently died from the disease (5-7). Due to advances in surgical techniques and chemotherapy, the mortality rate for bladder cancer has been declining over the past few decades, but the overall five-year survival rate did not significantly increase with the metastatic or advanced disease ranges from $13 \%$ to $15 \%$ (8). Therefore, searching novel biomarkers for prediction and prognosis of bladder cancer and new therapeutic targets is a major goal in this area (9).

The matrix metalloproteinases (MMPs) comprise a family of 23 structurally related and zinc-dependent endopeptidase which break down extracellular matrix and regulates the activity of cytokines and growth factors. Meanwhile, it can play a significant role in many physiological functions, such as development, inflammation, wound healing, and angiogenesis (10-13), and it has been shown in many literatures that the up-regulation of matrix metalloproteinases is related to the occurrence and development of tumors. 
MMP-28 (also called epilysin), structurally belong to the MMP-19 subfamily, more it has the archetypal domain of MMP and also contains a furan activation sequence, which is always activated in the secretory pathway. What's more, MMP-28 has been demonstrated localizes to the plasma membrane of cells and expressed in various normal and cancer tissues (14-16). In early pregnancy, MMP-28 is present in fetal tissue and rhesus monkey placenta. It was also prominently stained in basal keratinocytes both at and some distance from the wound edge during the wounded human skin and increased in demyelinating lesions in human multiple sclerosis (17-19). Hence, it's function in normal tissue and wound repair as well as in tumor progression is suggested $(15,18)$. MMP-28 has been found to be expressed in ovarian, pancreatic and colon carcinoma. Recently, it was shown in several tumor cell such as prostate carcinoma, lung carcinoma and so on (18).

Therefore, these findings suggest a central physiological role of MMP-28 in embryogenesis and tumor development, so we hypothesized that MMP-28 is a maker with prognostic value for cancer. In this work, in order to provide some further insight into the function of MMP-28, especially in the urinary system, we examined the expression and activation of MMP-28 and the situation of stable over-expression of the gene in bladder cancer cells.

\section{Methods}

\section{1 patient population and tissue samples}

From January 1998 to December 2011, A total of 491 bladder cancer tissue specimens were collected at Zhejiang Provincial People's Hospital (aged from 35 to 79 years old, median age 63.2 years old). All patients were followed up for more than 5 years and the deadline was December 2017. The survival time was calculated from the operation time to the follow-up deadline or the date of death. Meanwhile, the cause of death was mainly due to cancer metastasis or recurrence. 80 controls were obtained from tumor tissue more than $5 \mathrm{~cm}$ away beyond the edge of the tumor. The Samples were brought into a tissue microarray format. In short, core tissue biopsies ( $2 \mathrm{~mm}$ in diameter), representing the most invasive areas of each cancer, were obtained from the targeted region of each donor tissue block located within the recipient paraffin blocks (tissue array blocks) using tissue microarray instrument. In each block, 6 cases of non-cancerous bladder mucosa were used as internal controls. 4- $\mu \mathrm{m}$-thick continuous sections were cut from each tissue array block. The tissue sections were deparaffinized and rehydrated. Lymphangitic invasion is based on the presence of cancer cells in endothelial cells. Cancer cells that only invade the lumen of blood vessels are considered benign. Histopathological parameters were assessed according to the 2009 TNM classification and WHO classification. All patients signed written informed consent and we obtained the ethical approval from the ethics committee of Zhejiang Provincial People's Hospital.

\subsection{Immunohistochemistry and scoring}

Immunohistochemistry was used to study the changes of protein expression in 80 non-carcinomatous human bladder tissue samples and 491 human bladder cancer tissues. Briefly, slides in bake $60^{\circ} \mathrm{C}$ for 2 
hours followed by deparaffinization with xylene and rehydrated. The sections were soaked in the buffer of EDTA antigen extraction and microwaved for antigen retrieval, then treated with $3 \%$ hydrogen peroxide in methanol to inhibit the activity of endogenous peroxidase, followed by incubated with $1 \%$ bovine serum albumin to block nonspecific binding. Sections choose the mice anti- MMP28 (Santa Cruz Biotechnology Inc, Delaware Avenue, $\mathrm{CA}$ ) incubation in $4^{\circ} \mathrm{C}$ refrigerator overnight for 16 hours. After washing, tissue sections were treated with Envision (Rabbit, Dako, Denmark) which was applied for the secondary antibody and choose goat serum as the negative control group. Tissue sections were then counterstained with hematoxylin, dehydrated, and mounted.

The grade of immunostaining was evaluated and scored independently by 2 observers based on the intensity of staining who were not understood of the clinical and pathologic data. In the cases of discrepancy, consensus scores were chosen for evaluation. Staining intensity was graded according to the following standards: (no staining) $=0$, (weak staining =light yellow) $=1$, (moderate staining = yellowish brown) $=2$, (strong staining $=$ brown) $=3$ and distribution (less than 5 percentage $=0,6-26$ percentage $=1$, $26-50$ percentage $=2$, more than 51 percentage $=3$ ) and calculating the staining index. We evaluated the expression of MMP28 by determining the score of $0,1,2,3,4,6$ or 9 of staining index. Selecting the optimal truncation value as: score $\geq 4$ was used to define tumors with high MMP28 expression, and score $\leq 3$ predict low MMP28 expression.

\subsection{Statistical analysis}

The statistical analysis in this study was carried out by the Statistical Package of Social Sciences (SPSS V.22.0 for Windows). To assess the relationship between the MMP28's expression and the clinicopathological parameters of bladder cancer patients, the Student's test was used to analyze the measurement data and the categorical data processing were using the Fisher's exact test or $X^{2}$. Survival curves were evaluated using the Kaplan-Meier method, and the log rank test detects the difference between curves. The Cox proportional hazard regression model was used for multivariate survival analysis to evaluate the prognostic value of protein expression. Spearsman correlation method was used to determine the correlation coefficients between protein expression and clinicopathological results. All $\mathrm{P}$ values were bilateral and when it's less than 0.05 were considered to be statistically significant.

\section{Results}

\section{Overexpression of MMP28 in bladder cancer tissues}

The immunostaining of MMP28 was mainly located in the cytoplasm of the tumor cells (Figure 1). MMP28 protein was detected in 8 of $80(10.0 \%)$ human non-tumor mucosa; all samples expressed the protein at low levels. MMP28 protein was detected in 333 of $491(67.82 \%)$ cases, which was significantly higher than that in the non-tumorous bladder mucosa $(P<0.01)$. A graphical form of the score values of immunostaining of MMP28 expression in tissues was shown in Figure 1. 
Positive expression of MMP28 correlated with tumor size, number of tumors, histological grade, invasion depth, lymph node metastasis, vessel invasion and distant metastasis $(P<0.05)$, but it did not correlate with age and gender. Bladder cancer patients with more than $3 \mathrm{~cm}$ tumor size, multiple tumor, deep tumor invasion (T2-T4), lymph node metastasis, distant metastasis and high grade had significantly higher expression of MMP28 than those with less than $3 \mathrm{~cm}$ tumor size区single tumor, superficial tumor invasion (Ta-T1), without lymph node metastasis, without distant metastasis and low grade (Table 1). The Spearman correlation coefficient of MMP28 expression with tumor size, number of tumors, depth of invasion, lymph node metastasis, histological grade and distant metastasis of tumor were $0.143,0.152$, $0.133,0.204,0.162$ and $0.173(P<0.05)$, respectively.

\section{Survival analysis}

The mean survival time in patients with high expression of MMP28 was $35.65 \pm 1.47$ months, which was significantly lower than in patients with low expression of MMP28 (56.36 \pm 1.25 months, $\mathrm{P}<0.001)$. Kaplan-Meier survival statistics showed that high expression of MMP28 was associated with worse overall survival when compared to low expression of MMP28 ( $<<0.001$, Figure 2$)$. Factors with possible prognostic effects in bladder cancer were analyzed by multivariate Cox proportional hazard regression analysis. Statistical analysis showed that depth of invasion ( $P=0.028)$, lymph node metastasis $(P=0.037)$ and expression of MMP28 $(\mathrm{P}<0.001)$ were highly significant independent prognostic factors for survival in patients with bladder cancer. However, age, size of tumor, number of tumors, lymphovascular invasion, distant metastasis, and histological grade had no prognostic value (Table 2).

\section{Discussion}

As one of the most common malignancies of the urinary system, Bladder cancer remains with a poor prognosis although some progress has been made in diagnosis and treatment in recent years. Despite several markers have been studied for commercial use in bladder cancer, including bladder tumor antigen (BTA) and NMP-22, more markers are still in the preclinical stage, such as microsatellite analysis, telomerase and survivin $(11,12,20)$. Especially on NMP-22 test, as a widely used method, has been shown to be elevated in some benign disease or malignant disease other than bladder can also cause increased NMP-22 levels similar to bladder cancer. Moreover, some studies on NMP-22 have revealed that the overall sensitivity and specificity of NMP-22 are low, which greatly limited its clinical application (21). Therefore, finding better, reliable and non-invasive bladder cancer markers for early diagnosis and postoperative review is a significant topic in urosurgery.

MMPs is a family of metal-dependent extracellular proteases, which are not generally expressed in normal tissues, their production and activity are upregulated during injury, inflammation, and tumorigenesis (22). However囚MMP-28 is unlike many traditional MMPs it was also expressed in normal tissues, including the basal and suprabasal keratinocytes of the skin, in developing testicular germ cells, and the lung $(15,18)$, its overexpression in carcinomas may predict survival in patients such as 
glioblastoma (23) and gastric cancer (24). In spite of this, MMP-28 and clinicopathological parameters have not been reported in bladder cancer yet.

Up to now, invasion depth, lymph node metastasis and distant metastasis are considered as prognostic factors of bladder cancer. According to our study, we have found that overexpression of MMP-28 was significantly related to poor prognosis of patients with bladder cancer, and MMP-28 was shown to be an independent prognostic. Further analysis showed that the mean survival time of patients with low MMP28 expression was significantly longer than that of patients with MMP-28 overexpression. It is suggested that MMP-28 can be used as an effective and objective indicator to identify the high-risk group of bladder cancer invasion and progression.

In the present study, immunohistochemistry was adopted to explore the expression of MMP-28 and its relationship with clinicopathological characteristics and prognosis in 491 patients with bladder cancer. Immune reaction scoring results of MMP-28 staining proved that MMP-28 was obviously increasing compared with that in normal bladder tissues, which indicated an important marker role of MMP-28 in bladder cancer. When analyzed the association of MMP-28 staining with clinicopathological and genetic features, we found that positive MMP-28 staining was more likely to be detected in large tumor size, multiple tumors and high histological grade, indicating the more malignant the tumor is in situ, the higher expression of MMP-28. Moreover, it's also strongly correlated with tumor metastasis. In our research, invasion depth, lymph node metastasis, vessel invasion and distant metastasis were also found to be highly expressed. However, MMP-28 expression was not found to be related with patients' age or gender.

As MMP-28 has been found that it can induce epithelial cells to transform into mesenchymal cells and invade cells through TGF- $\beta$-dependent mechanism, thus promoting the progress of bladder cancer. Therefore, we analyzed the prognostic value of MMP-28 on the overall survival of bladder cancer patients. Kaplan-Meier analysis revealed a statistically significant correlation between increased MMP-28 expression and poor overall survival in bladder cancer patients, showing that patients with bladder cancer of positive MMP-28 staining had a higher risk of death compared with those with negative MMP-28 staining. Besides, elevated MMP- 28 expression had shorter overall survival time than those with low expression, and the prognostic effect of MMP-28 expression was independent prognostic elements for bladder cancer.

\section{Conclusions}

In summary, our study had demonstrated the overexpression of MMP-28 was related to overall survival of bladder cancer, which suggest that the capacity of MMP-28 to predict poor prognosis and help us understand more molecular oncogene mechanisms in urothelial carcinoma. At the same time, based on the TNM stage, the expression of MMP-28 in bladder cancer will also help to identify patients with high potential for tumor metastasis, which predicting the prognosis of the patients with urinary bladder cancer. Thus, MMP-28 may play a significant role in the progression of bladder cancer and could be an effective indicator to predict tumor invasion and prognosis for bladder cancer. 


\section{Abbreviations}

BC: Bladder Cancer; MMP: Matrix Metalloproteinases

\section{Declarations}

\section{Ethics approval and consent to participate}

The experimental protocol was established, according to the ethical guidelines of the Helsinki Declaration and was approved by the Human Ethics Committee of Zhejiang Provincial People's Hospital.

\section{Consent for publication}

Not applicable

\section{Availability of data and material}

All data generated or analyzed during this study are included in this published article and its supplementary information files.

\section{Competing interests}

The authors declare that they have no competing interests.

\section{Funding}

This study was supported in part by grants from natural science foundation of Zhejiang province (GF19H160084) and Zhejiang traditional Chinese medicine administration(2017ZZ002).

\section{Authors' contributions}

Qi Zhang analyzed and interpreted the patient data regarding the bladder cancer. Jun-xiu Wu performed the histological examination of the tissue, and Heng Wang was a major contributor in writing the manuscript. All authors read and approved the final manuscript.

\section{Acknowledgements}

Not applicable.

\section{References}

1. S. C. Skeldon, S. Larry Goldenberg, Bladder cancer: a portal into men's health. Urol Onco/ 33, 40-44 (2015).

2. G. L. Yang et al., Increased expression of HMGB1 is associated with poor prognosis in human bladder cancer. Journal of surgical oncology 106, 57-61 (2012). 
3. G. Dalbagni, The management of superficial bladder cancer. Nature clinical practice. Urology 4, 254260 (2007).

4. L. Xia, T. J. Guzzo, Preoperative Anemia and Low Hemoglobin Level Are Associated With Worse Clinical Outcomes in Patients With Bladder Cancer Undergoing Radical Cystectomy: A Meta-Analysis. Clin Genitourin Cancer 15, 263-272.e264 (2017).

5. R. M. Anghel, L. N. Gales, O. G. Trifanescu, Outcome of urinary bladder cancer after combined therapies. Journal of medicine and life $9,153-159$ (2016).

6. S. A. Hussain, N. D. James, The systemic treatment of advanced and metastatic bladder cancer. The Lancet. Oncology 4, 489-497 (2003).

7. Y. J. Xue, Q. Lu, Z. X. Sun, CD147 overexpression is a prognostic factor and a potential therapeutic target in bladder cancer. Medical oncology (Northwood, London, England) 28, 1363-1372 (2011).

8. A. J. Cohen et al., Upstaging of nonurothelial histology in bladder cancer at the time of surgical treatment in the National Cancer Data Base. Urol Onco/ 35, 34.e31-34.e38 (2017).

9. Q. Zhang et al., Upregulated expression of annexin II is a prognostic marker for patients with gastric cancer. World journal of surgical oncology 10, 103 (2012).

10. M. A. Arnaout, S. L. Goodman, J. P. Xiong, Structure and mechanics of integrin-based cell adhesion. Current opinion in cell biology 19, 495-507 (2007).

11. B. K. Pilcher et al., Role of matrix metalloproteinases and their inhibition in cutaneous wound healing and allergic contact hypersensitivity. Annals of the New York Academy of Sciences 878, $12-24$ (1999).

12. S. F. Shariat et al., Urine detection of survivin is a sensitive marker for the noninvasive diagnosis of bladder cancer. J Uro/ 171, 626-630 (2004).

13. M. J. Wallard et al., Comprehensive profiling and localisation of the matrix metalloproteinases in urothelial carcinoma. British journal of cancer 94, 569-577 (2006).

14. S. A. Illman, J. Keski-Oja, W. C. Parks, J. Lohi, The mouse matrix metalloproteinase, epilysin (MMP28), is alternatively spliced and processed by a furin-like proprotein convertase. The Biochemical journal 375, 191-197 (2003).

15. J. Lohi, C. L. Wilson, J. D. Roby, W. C. Parks, Epilysin, a novel human matrix metalloproteinase (MMP28) expressed in testis and keratinocytes and in response to injury. J Biol Chem 276, 10134-10144 (2001).

16. A. M. Manicone et al., Matrix Metalloproteinase-28 Is a Key Contributor to Emphysema Pathogenesis. The American journal of pathology 187, 1288-1300 (2017).

17. Q. L. Li et al., Matrix metalloproteinase-28 transcript and protein are expressed in rhesus monkey placenta during early pregnancy. Molecular human reproduction 9, 205-211 (2003).

18. G. N. Marchenko, A. Y. Strongin, MMP-28, a new human matrix metalloproteinase with an unusual cysteine-switch sequence is widely expressed in tumors. Gene 265, 87-93 (2001). 
19. S. R. Werner, J. E. Dotzlaf, R. C. Smith, MMP-28 as a regulator of myelination. BMC neuroscience $\mathbf{9}$, 83 (2008).

20. S. F. Shariat et al., Multiple biomarkers improve prediction of bladder cancer recurrence and mortality in patients undergoing cystectomy. Cancer 112, 315-325 (2008).

21. S. F. Shariat et al., Variability in the performance of nuclear matrix protein 22 for the detection of bladder cancer. J Uro/ 176, 919-926; discussion 926 (2006).

22. H. Nagase, J. F. Woessner, Jr., Matrix metalloproteinases. J Biol Chem 274, 21491-21494 (1999).

23. X. Wang, K. Zhang, X. Chen, C. Zhao, Z. Sun, Epilysin is overexpressed in glioblastoma and related to clinical outcome of patients. Medical oncology (Northwood, London, England) 32, 363 (2015).

24. J. Zhang et al., Overexpression of MMP21 and MMP28 is associated with gastric cancer progression and poor prognosis. Oncology letters 15, 7776-7782 (2018).

\section{Tables}

Table 1. Relationship of MMP28 expression with pathological parameters of bladder cancer 


\begin{tabular}{|c|c|c|c|c|}
\hline \multirow[t]{2}{*}{ Clinical parameters } & \multicolumn{4}{|c|}{ MMP28 expression } \\
\hline & Low & High & c2 & $P$ \\
\hline Gender & & & 0.091 & 0.763 \\
\hline Male & $138(87.3 \%)$ & 294 (88.3\%) & & \\
\hline Female & $20(12.7 \%)$ & 39 (11.7\%) & & \\
\hline Age(yrs) & & & 0.187 & 0.665 \\
\hline$<60$ & $58(36.7 \%)$ & $129(38.7 \%)$ & & \\
\hline$\geq 60$ & $100(63.3 \%)$ & $204(61.3 \%)$ & & \\
\hline Size & & & 10.102 & 0.001 \\
\hline$<3 \mathrm{~cm}$ & 94 (59.5\%) & $147(44.1 \%)$ & & \\
\hline$\geq 3 \mathrm{~cm}$ & $64(40.5 \%)$ & $186(55.9 \%)$ & & \\
\hline Number of tumor & & & 11.397 & 0.001 \\
\hline single & $47(29.7 \%)$ & $55(16.5 \%)$ & & \\
\hline multiple & $111(70.3 \%)$ & 278 (83.5\%) & & \\
\hline Invasion depth & & & 8.632 & 0.003 \\
\hline Тa-T1 & $32(20.3 \%)$ & 35 (10.5\%) & & \\
\hline T2-T4 & $126(79.7 \%)$ & $298(89.5 \%)$ & & \\
\hline Lymph node metastasis & & & 20.551 & 0.000 \\
\hline No & $139(88.0 \%)$ & $230(69.1 \%)$ & & \\
\hline Yes & $19(12.0 \%)$ & $103(30.9 \%)$ & & \\
\hline Distant metastasis & & & 14.740 & 0.000 \\
\hline No & $156(98.7 \%)$ & $295(88.6 \%)$ & & \\
\hline Yes & $2(1.3 \%)$ & $38(11.4 \%)$ & & \\
\hline Lymphovascular invasion & & & 5.459 & 0.019 \\
\hline negative & $151(95.6 \%)$ & 297 (89.2\%) & & \\
\hline positive & $7(4.4 \%)$ & $36(10.8 \%)$ & & \\
\hline Histological grade & & & 25.755 & 0.000 \\
\hline Low grade (superficial) & $68(43.1 \%)$ & $99(29.7 \%)$ & & \\
\hline High grade (infiltrating) & $90(57.0 \%)$ & $234(70.3 \%)$ & & \\
\hline
\end{tabular}

Table 2 Multivariate analysis of the correlation between clinicopathological parameters and survival time of patients with bladder cancer 


\begin{tabular}{|c|c|c|c|c|c|}
\hline Covariates & Coefficient & $\begin{array}{c}\text { Standard } \\
\text { error }\end{array}$ & $\begin{array}{c}\text { Hazard } \\
\text { ratio } \\
(\mathrm{HR})\end{array}$ & $\begin{array}{l}95.0 \% \mathrm{Cl} \\
\text { for HR }\end{array}$ & $P$ \\
\hline Age range (>60 vs $\leq 60)$ & 0.041 & 0.202 & 1.042 & $0.701-$ & 0.838 \\
\hline Tumor size $(\geq 3 \mathrm{~cm}$ vs $<3 \mathrm{~cm}$ ) & -0.056 & 0.143 & 0.946 & $0.715-$ & 0.697 \\
\hline Number of tumor (single vs multiple) & 0.108 & 0.177 & 1.114 & $0.788-$ & 0.541 \\
\hline $\begin{array}{l}\text { Lymph node metastasis (positive vs } \\
\text { neqative) }\end{array}$ & 0.336 & 0.161 & 1.399 & $\begin{array}{l}1.020- \\
1.917\end{array}$ & 0.037 \\
\hline $\begin{array}{l}\text { Lymphovascular invasion (positive vs } \\
\text { negative) }\end{array}$ & -0.020 & 0.253 & 0.980 & $\begin{array}{l}0.597- \\
1.608\end{array}$ & 0.980 \\
\hline Distant metastasis (positive vs negative) & 0.370 & 0.252 & 1.448 & $0.883-$ & 0.142 \\
\hline MMP28 expression (high vs low) & 0.888 & 0.165 & 2.429 & $1.758-$ & 0.000 \\
\hline Depth of invasion ( T2-T4 vs Ta-T1) & 0.324 & 0.148 & 1.383 & $1.036-$ & 0.028 \\
\hline Histological grade & -0.039 & 0.122 & 0.962 & $\begin{array}{l}0.757- \\
1.223\end{array}$ & 0.753 \\
\hline
\end{tabular}

\section{Figures}

\section{Figure 1}

Immunohistochemical staining for MMP-28 in normal and cancerous bladder tissue. A1-A3:

Immunostaining of MMP-28 in normal tissue. B1-B3: strong staining (yellow-brown granules, mainly in the cytoplasm) in nonmuscle-invasive urinary bladder carcinoma. C1-C3: Strong staining in muscleinvasive urinary bladder carcinoma. Magnification: the original magnification x40 (A1-C1), x100 (A2-C2), and $\mathrm{x} 400$ (A3-C3). 


\section{Survival Functions}

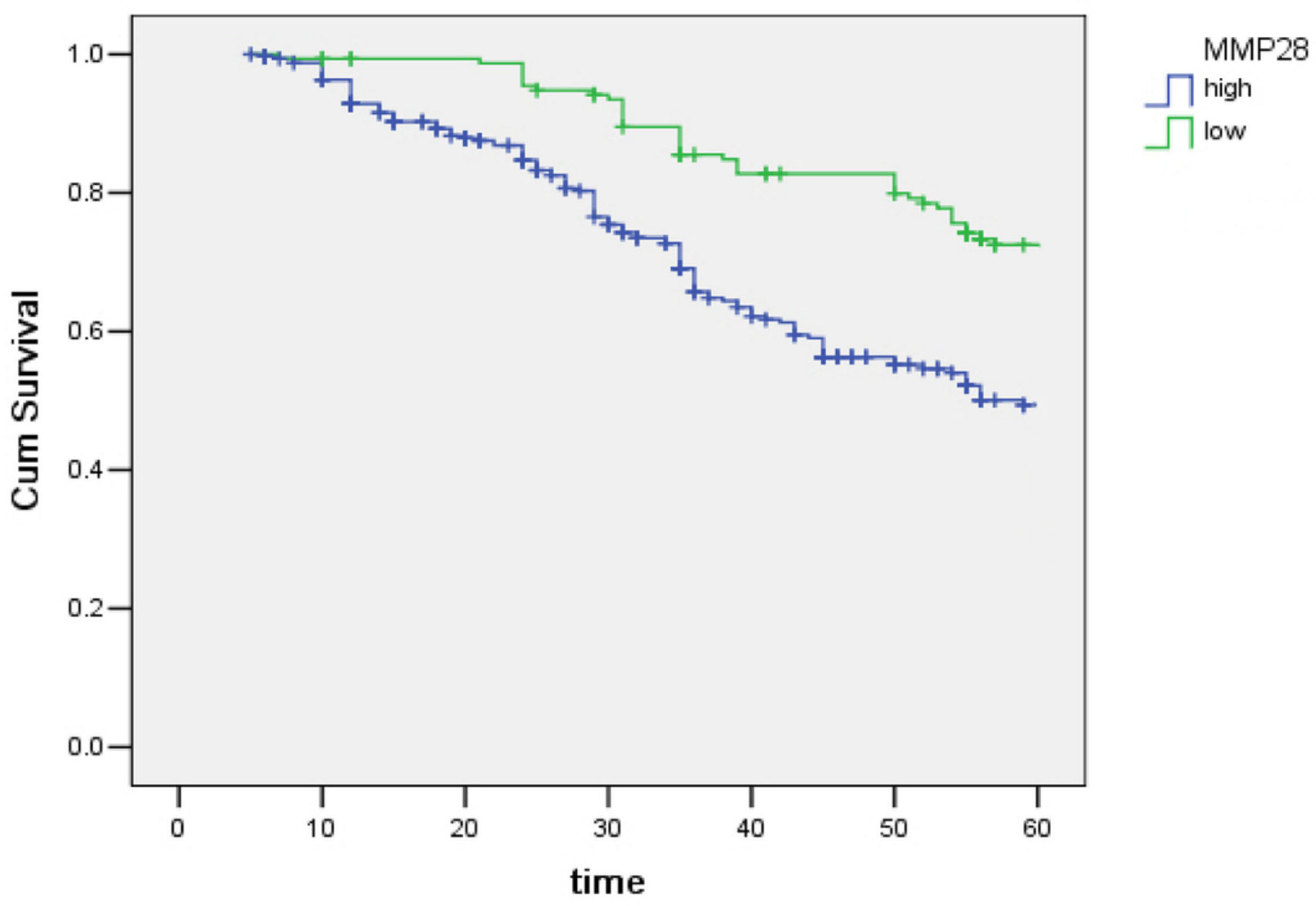

Figure2. Kaolan-Meier survival curves of urinary bladder carcinoma patients with positive and negative MMP-28 expression.

Figure 2

Kaolan-Meier survival curves of urinary bladder carcinoma patients with positive and negative MMP-28 expression 